\title{
Assessing the beginning to end-of-mission sensitivity change of the PREcision MOnitor Sensor total solar irradiance radiometer (PREMOS/PICARD)
}

\author{
William T. Ball, Werner Schmutz, André Fehlmann, ${ }^{\text {, }}$, Wolfgang Finsterle, and Benjamin Walter \\ Physikalisch-Meteorologisches Observatorium Davos World Radiation Centre, Dorfstrasse 33, 7260 Davos Dorf, Switzerland \\ ${ }^{a}$ Current address: Institute for Astronomy, University of Hawaii, 34 Ōhi'a Kū St. Pukalani, HI 96768-8288, USA \\ ${ }^{*}$ Corresponding author: william.ball@pmodwrc.ch
}

Received 2 February 2016 / Accepted 1 July 2016

\begin{abstract}
The switching of the total solar irradiance (TSI) backup radiometer (PREMOS-B) to a primary role for 2 weeks at the end of the PICARD mission provides a unique opportunity to test the fundamental hypothesis of radiometer experiments in space, which is that the sensitivity change of instruments due to the space environment is identical for the same instrument type as a function of solar-exposure time of the instruments. We verify this hypothesis for the PREMOS TSI radiometers within the PREMOS experiment on the PICARD mission. We confirm that the sensitivity change of the backup instrument, PREMOS-B, is similar to that of the identically-constructed primary radiometer, PREMOS-A. The extended exposure of the backup instrument at the end of the mission allows for the assessment, with an uncertainty estimate, of the sensitivity change of the primary radiometer from the beginning of the PICARD mission compared to the end, and of the degradation of the backup over the mission. We correct six sets of PREMOS-B observations connecting October 2011 with February 2014, using six ratios from simultaneous PREMOS-A and PREMOS-B exposures during the first days of PREMOS-A operation in 2010. These ratios are then used, without indirect estimates or assumptions, to evaluate the stability of SORCE/TIM and SOHO/VIRGO TSI measurements, which have both operated for more than a decade and now show different trends over the time span of the PICARD mission, namely from 2010 to 2014. We find that by February 2014 relative to October 2011 PREMOS-B supports the SORCE/TIM TSI time evolution, which in May 2014 relative to October 2011 is $\sim 0.11 \mathrm{~W} \mathrm{~m}^{-2}$, or $\sim 84 \mathrm{ppm}$, higher than SOHO/VIRGO. Such a divergence between SORCE/TIM and SOHO/VIRGO over this period is a significant fraction of the estimated decline of $0.2 \mathrm{~W} \mathrm{~m}^{-2}$ between the solar minima of 1996 and 2008, and questions the reliability of that estimated trend. Extrapolating the uncertainty indicated by the disagreement of SORCE/TIM and PREMOS with respect to SOHO/VIRGO, we can conclude that it is currently not possible to assess centennial timescale changes in solar irradiance based on any of the presently existing TSI composites. It is imperative to accurately estimate solar irradiance changes from observations in order to extrapolate centennial scale trends important for understanding both long-term solar irradiance changes and the Sun's influence on the Earth's climate.
\end{abstract}

Key words. Total solar irradiance - Observations - Radiometry - Space mission - PREMOS - PICARD

\section{Introduction}

A high precision and accurate measurement record of the spectrally-integrated, total solar irradiance (TSI) arriving at the top of the atmosphere is essential for understanding both the energy balance of the Earth's climate system and the impact of TSI variations on decadal and centennial timescales. These timescales are essential to understand the relative contribution of solar variability to recent estimates of anthropogenic, and future, climate change.

Stitching together TSI instrument records leads to large uncertainties based on how data are combined (Willson \& Mordvinov 2003; Dewitte et al. 2004; Fröhlich 2013). The PMOD composite suggests that there was a decline of $0.2 \mathrm{~W} \mathrm{~m}^{-2}$ between 1996 and 2008 (Fröhlich 2009), though the uncertainty is of a similar magnitude and it is, therefore, not currently possible to be confident of centennial trends in TSI. Of the 12-or-so instruments that have acquired TSI measurements since 1978 (Kopp 2014), the only instrument with continuous daily observations covering changes between solar cycle minima is the Variability of solar IRradiance and Gravity Oscillations (VIRGO) experiment (Fröhlich et al. 1997) on the Solar and Heliospheric Observatory (SOHO). The stability of SOHO/VIRGO is remarkably good for a space experiment with such an unusually long flight duration. Fröhlich (2016, personal communication) estimates the stability of his composite to be $70 \mathrm{ppm}$ per cycle, or $6 \mathrm{ppm} \mathrm{yr}^{-1}$, whereas Kopp (2014) estimates the long-term uncertainty of

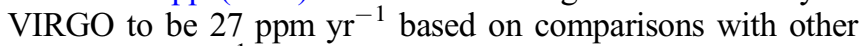
TSI instruments. ${ }^{1}$

Kopp (2014) proposes that, for judging the climate-relevant impact of TSI, an accuracy better than $0.001 \% \mathrm{yr}^{-1}$

\footnotetext{
${ }^{1}$ We note that there was a change of SOHO/VIRGO data release versions from v6_004_1512 to v6_005_1602 during the preparation of this article. This change led to a difference in the change between October/November 2011 and April/May 2014 of $0.015 \mathrm{~W} \mathrm{~m}^{-2}$, or $11 \mathrm{ppm} \mathrm{yr}^{-1}$. This modification alone suggests that the stability of $\mathrm{SOHO} /$ VIRGO over this period is at least nearly double the $6 \mathrm{ppm}$ estimate. A similar conclusion was reached by Kopp (2016).
} 


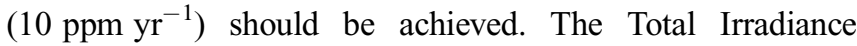
Monitor (TIM), launched in 2003 on the Solar Radiation and Climate Experiment (SORCE) satellite, with an estimated stability of $10 \mathrm{ppm} \mathrm{yr}^{-1}$, is considered by the community to be the most stable TSI instrument launched to date. By comparing the records of the SOHO/VIRGO and SORCE/TIM instruments, we notice a trend between them of $\sim 15 \mathrm{ppm} \mathrm{yr}^{-1}$ from 2003 to 2013, a diverging trend that has accelerated since 2008. The difference between these experiments has reached $\sim 0.28 \mathrm{~W} \mathrm{~m}^{-2}$, or $\sim 200 \mathrm{ppm}$, by June-August 2014 , relative to September-November 2003. Another instrument, the Active Cavity Radiometer Irradiance Monitor III (ACRIM-III; Willson 2014), has had a trend that diverges from SORCE/ TIM by at least $33 \mathrm{ppm} \mathrm{yr}^{-1}$ over the same period (Kopp 2014), but we do not consider it here as the mission ended in December 2013. It appears that instruments are not accurate enough over decadal timescales to determine if multi-decadal variations in TSI exist or, if they do, how large they might be. Over the period from 2011 to 2014, during which the PREcision MOnitor Sensor (PREMOS) TSI mission operated and considered in this study, SORCE/TIM and SOHO/VIRGO diverged by $\sim 0.11 \mathrm{~W} \mathrm{~m}^{-2}$. Space-based TSI instruments are subject to degradation, which requires some assumption about the sensitivity change of the backup channel, which is used to determine the relative in-flight long-term irradiance calibration. Here, we use the measured degradation of the PREMOS-A radiometer to correct the backup radiometer PREMOS-B and, subsequently, infer whether SORCE/TIM or SOHO/VIRGO is more likely to have reported the correct TSI changes during the 2011-2014 period.

\section{Instrument degradation}

In-flight change of a cavity's sensitivity is typical for radiometers. For example, the primary radiometer of SORCE/TIM experienced $\sim 200 \mathrm{ppm}$ degradation over the first 10 years of operation (2003-2012; Kopp 2014). PMO6 radiometers, which comprise both PREMOS radiometers and one half of the $\mathrm{SOHO}$ /VIRGO experiment (see below), can exceed thousands of ppm per decade (Fröhlich 2003; Schmutz et al. 2013). For example, the A-sensor of the PREMOS TSI radiometer on the PICARD satellite, "PREMOS-A", degraded by almost $3,000 \mathrm{ppm}$ in 2 years of operation, during which it was exposed for 300 days (Fehlmann et al. 2012; Schmutz et al. 2013). This is why all TSI radiometers in space, other than the Hickey-Frieden (HF; Hickey et al. 1988) and Earth Radiation Budget Satellite (ERBE; Lee et al. 1995) experiments, had a backup channel in order to account for the degradation in the operating instrument (SORCE/TIM has three; Kopp \& Lawrence 2005). The backup radiometer is exposed only infrequently and thus degrades only fractionally relative to the primary operational radiometer. Intermittent comparisons of the primary cavity with this more stable backup throughout the instrument's lifetime enable degradation in the primary to be monitored and thus corrected. The caveat is that there is also degradation in the backup radiometer, and the ability to correct the primary channel requires an estimation of how both the primary and the backup radiometers have degraded over the entire mission period.

The basic hypothesis of space radiometry is that one can use the sensitivity changes of the primary to estimate that of the secondary as a function of solar-exposure time or, more correctly formulated, as a function of dose. "Exposure time", measured in units of exposure days, means the accumulated time for which the cavity is directly exposed to the Sun; so for one observation cycle of $2 \mathrm{~min}$, the active cavity on PREMOS will accumulate one additional minute of exposure, with the cavity closed for the other minute. The accumulated "dose" of the observing instrument represents the accumulated damaging radiation flux admitted into the cavity, assumed to be due to high-energy ultraviolet radiation (Fröhlich 2003); the dose is approximately related to exposure time by the activity level of the Sun during each exposure. To first order the length of time exposed is a good approximation for the degradation, and accounting for dose requires some assumptions as to which particular wavelengths of solar radiation lead to the instrument degradation. We have evaluated the impact of weighting the dose by the variations of Lyman- $\alpha$, which is an extreme modification of a realistic dose. With this extreme estimate we verify that the way we evaluate the dose does not have an impact on the conclusion of our investigation.

Up to the end-of-mission it had been difficult to know if the PREMOS TSI backup instrument had degraded in exactly the same way as the primary, and this introduces additional uncertainties. $^{2}$ PREMOS comprises the primary and backup radiometers: "PREMOS-A" and "PREMOS-B". These are PMO6-type radiometers, similar in design to the PMO6-V detectors that have been used in the PMOD instrument on VIRGO, which we call hereafter the PMO6-V instrument. The PMO6-V radiometer was one of the two instruments that comprise the SOHO/VIRGO package; the other VIRGO radiometer was the Differential Absolute Radiometer (DIARAD; Dewitte et al. 2004), which has a different design. In PMO6-type radiometers, there is an initially fast, linear increase in sensitivity for the first few days of exposure before a large exponential decline dominates (see Fröhlich 2003; Schmutz et al. 2013). The cause of this behaviour is thought to be the degradation of the absorptivity, on one hand in the measuring cavity, and on the other hand in the baffle system between the view limiting aperture and the precision aperture. The former process accounts for degradation of the instrument's sensitivity, the latter for an increase of the scattered light contribution that reaches the cavity.

Correcting a double cavity TSI experiment to better than $10 \mathrm{ppm} \mathrm{yr}^{-1}$ is still a difficult task in practice. Nevertheless, Schmutz et al. (2013) and Kopp (2014) reported agreement to within $5 \mathrm{ppm}$ between PREMOS/PICARD and SORCE/ TIM over the period 2010-2012. Over this period, SOHO/ VIRGO also agreed well with SORCE/TIM, but from mid-2012 SORCE/TIM diverged from SOHO/VIRGO and, by mid-2014 relative to late-2011, the two instruments differed by $\sim 0.114 \mathrm{~W} \mathrm{~m}^{-2}$ (Fig. 1). The PREMOS data release version 1 (Schmutz et al. 2013) has an intermediate behaviour from September 2012 to January 2014, with agreement changing between SORCE/TIM and SOHO/VIRGO (not shown). However, version 1 of the PREMOS TSI data release was an assessment until 2012 and the determination of the sensitivity change of the PREMOS experiment after 2012 is still work in progress. Here we present a direct assessment of the sensitivity change of the PREMOS-B radiometer between beginning and end-of-mission.

\footnotetext{
${ }^{2}$ Typically, PREMOS-A is exposed for $60 \mathrm{~s}$ every second minute and, apart from periods of switch off, this occurred for the duration over the mission up to the switch to PREMOS-B as the primary radiometer. As backup, PREMOS-B was continuously active once every 14 days for $96 \mathrm{~min}$, which is one PICARD orbit (i.e. it was exposed to sunlight for $48 \mathrm{~min}$ ). During PREMOS-B's period as primary, PREMOS-A was exposed as backup on two occasions.
} 


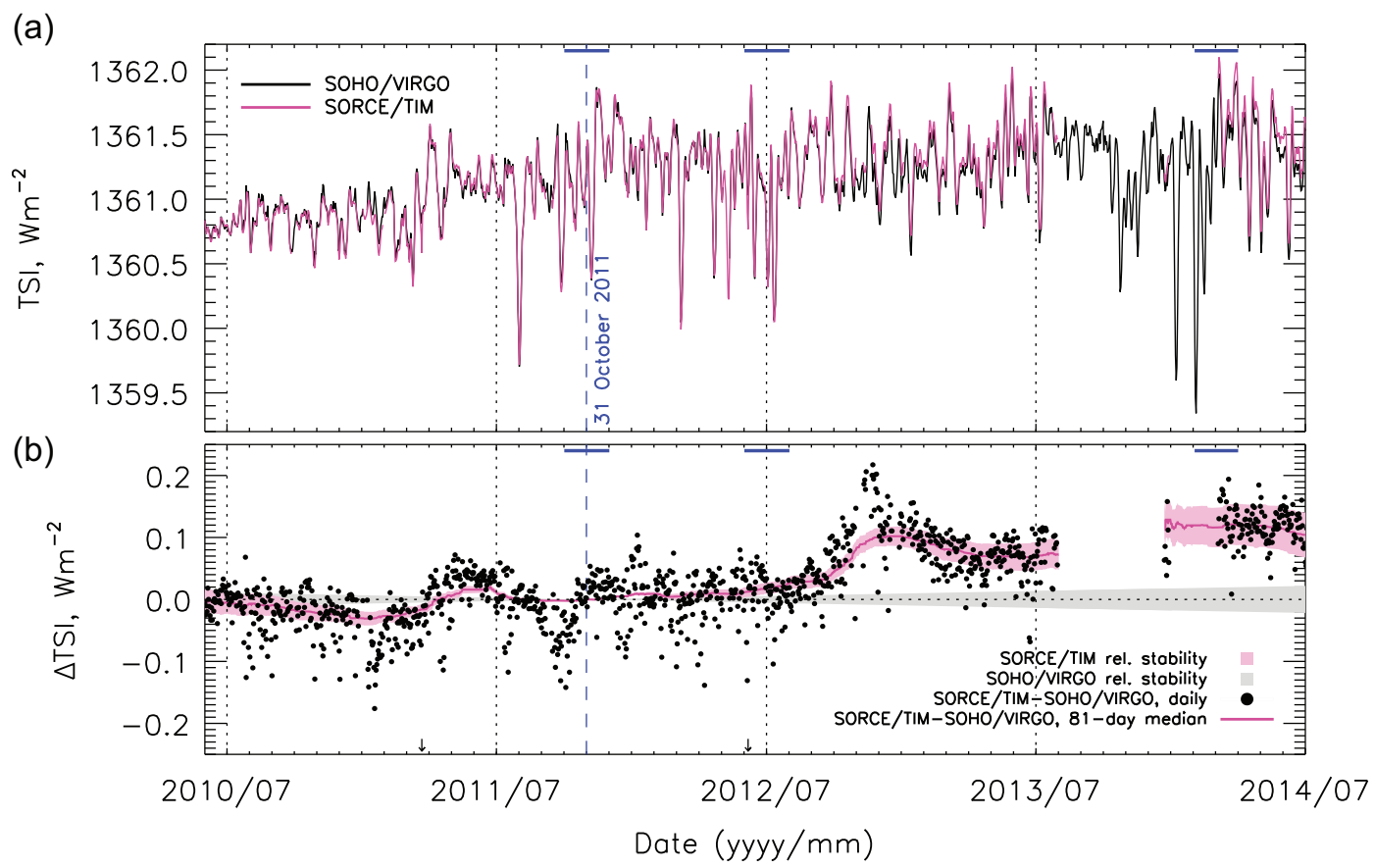

Fig. 1. (a) Absolute TSI observed by VIRGO/SOHO (black) and SORCE/TIM (purple) between June 2010 and July 2014. SOHO/VIRGO is shifted by $+0.05 \mathrm{Wm}^{-2}$ to agree with SORCE/TIM on 31 October 2011 (dashed vertical blue line), which is the reference date for comparison with PREMOS-B. (b) TSI of SORCE/TIM minus VIRGO/SOHO (daily, black dots; smoothed, purple line). SORCE/TIM smoothed and daily data have been shifted so that the median line crosses the zero line. The uncertainty on smoothed SORCE/TIM (pink) is $10 \mathrm{ppm}^{\mathrm{yr}}{ }^{-1}$, and $6 \mathrm{ppm} \mathrm{yr}^{-1}$ for SOHO/VIRGO (grey, along the $\Delta \mathrm{TSI}=0$ dotted line; see Footnote 1) relative to 31 October 2011. The blue horizontal bars represent the periods plotted in Figure 4.

In Figure 2 we show the ratio of PREMOS-A to DIARAD (blue dots) and to PMO6-V (yellow dots) for the first 14 exposure days of PREMOS-A ( $\sim$ July and August 2010; see also Figs. $3 \mathrm{a}$ and $3 \mathrm{~b}$ ). For PREMOS-A and -B we use averages of 2-minute cadence measurements, which fall within periods covered by the hourly data of the individual VIRGO instruments DIARAD and PMO6-V (which are published on the VIRGO website ${ }^{3}$ ). For PREMOS-A exposure days 0 to 4 , there is an apparent quasi-linear increase with respect to both VIRGO radiometers though, prior to 2.1 exposure days, the scatter of points between PREMOS-A and DIARAD is far smaller than for PREMOS-A:PMO6-V. Between 2.1 and 5.6 exposure days (between the grey vertical lines), an additional PREMOS instrument heater was switched on (see also grey dots between days 42 and 50 in Fig. 3b); it was switched off after it was clear this was causing enhanced scatter in the PREMOS-A observations. If we consider 6-h averages (darkgrey circles), the scatter shrinks and the sensitivity increase of PREMOS-A appears linear. After 5.6 exposure days, when the heater was switched off, the scatter of the PREMOSA:DIARAD ratio is equal to that from the first 2.1 exposure days. From about exposure day 4, it is clear that the quasilinear sensitivity increase of PREMOS-A ends and enters a period in which cavity degradation begins to dominate. Peak sensitivity is reached at day 7 , and it declines thereafter.

PREMOS-B became the primary instrument on 12 February 2014 after it had accumulated 3.75 exposure days (black vertical line in Fig. 2; see also Fig. 3c) which corresponds to 1,296 days after PREMOS switch on (see

\footnotetext{
${ }^{3}$ We use VIRGO, DIARAD and PMO6-V data version 42_65_1602. The data can be downloaded at https://www. pmodwrc.ch/pmod.php?topic=tsi/virgo/proj_space_virgo
}

Fig. 3b). PREMOS-B was then operated continuously as the primary instrument until 21 February and then again from 23 February until 4 March 2014. From the ratio of PREMOS-B to DIARAD (red dots in Fig. 2), it is clear that PREMOS-B underwent a practically identical degradation behaviour as PREMOS-A did for the same exposure interval (compare with blue dots of PREMOS-A:DIARAD). Apart from a possibly slightly different behaviour in the DIARAD reference, a small difference in the time evolution of the exposure ratios of PREMOS-A and B may be attributed to different levels of accumulated radiation during this period of exposure time, with PREMOS-A being exposed during low-medium solar activity in 2010 compared to higher activity for PREMOS-B in 2014, and thus PREMOS-B should have received a higher dose of degrading ultraviolet radiation during this time. However, for our purpose this difference is so small that we ignore it and assume the dose to be proportional to exposure days for 2010 and 2014 (see also Sect. 4). The comparison of these measurement-ratios is highly suggestive that the assumption that the first few exposure days of PREMOS-B follow the same sensitivity change behaviour as PREMOS-A is valid.

One cannot use the ratio of PREMOS-B to DIARAD as a valid test of the hypothesis for exposure less than 3.75 days because this period is influenced by 3 years of unknown long-term stability of the DIARAD radiometer. However, the ratios PREMOS-A:PREMOS-B, labelled 1-6 in Figure 3a with the dates listed in Table 1, display the same behaviour as the ratios PREMOS-A:DIARAD and PREMOS-A:PMO6-V at the beginning of the mission in 2010 (Fig. 2). The consistent behaviour of PREMOS-A and PREMOS-B, with respect to the other instruments, provides high confidence that PREMOS-B has degraded in the same way as PREMOS-A. 


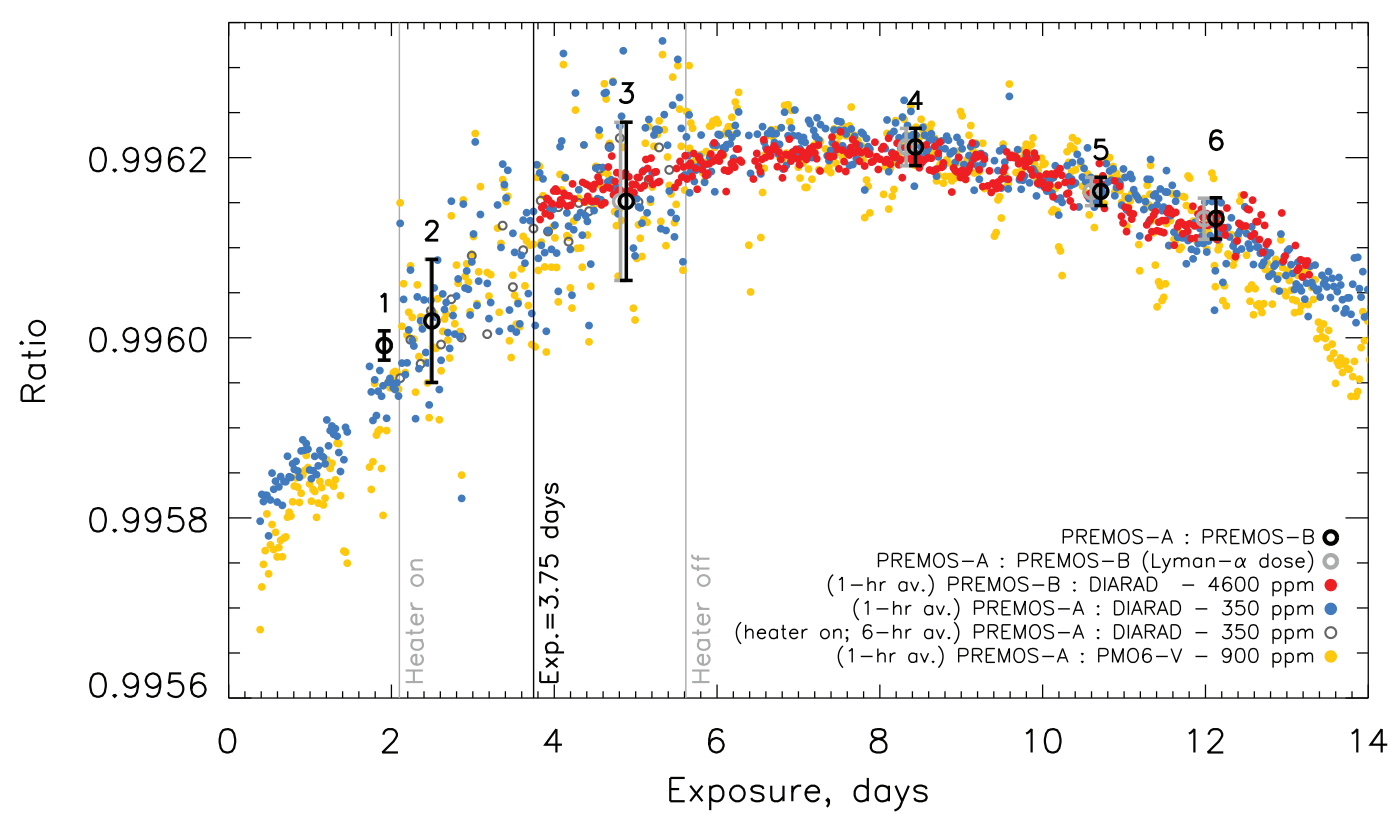

Fig. 2. The ratio PREMOS-A:PREMOS-B (thick black circles with error bars, labelled 1-6), as plotted in Figure 3 a, with hourly average ratios when there is overlap for PREMOS-A:PMO6-V/SOHO and PREMOS-A:DIARAD/SOHO (yellow and blue dots, respectively). Six-hourly averages for PREMOS-A:DIARAD/SOHO (dark-grey circles) are given for the period between the grey vertical lines labelled "Heater on" and "Heater off". PREMOS-B:DIARAD (red dots) is plotted after 3.746 days of PREMOS-B exposure. Exposure time is applicable to PREMOS-A or PREMOS-B, depending on which is the numerator. All ratios are shifted by the value indicated in the legend. Thick grey circles with error bars represent an exposure time estimate based on Lyman- $\alpha$ (see Sect. 4).

As a side note, we report that the ratio of PREMOS-B to DIARAD in 2014 has an approximately Gaussian scatter with one standard deviation of 12 ppm about the trend (red dots in Fig. 2). Assuming equal contributions by the two instruments, this implies an $8.5 \mathrm{ppm}$ precision for their 1-h TSI averages. A similar estimate holds for the ratio of PREMOS-A to DIARAD (blue dots in Fig. 2), except for the period when the additional heater was switched on, at the beginning of the PREMOS measurements in 2010.

\section{Long-term sensitivity corrections}

In the previous section we have verified that PREMOS-B has followed the same sensitivity behaviour as PREMOS-A. We can use the ratios PREMOS-A:PREMOS-B $1-6$ to compute ratios of PREMOS-A sensitivity changes when we include the very small PREMOS-B sensitivity changes during the first days of the mission. These are not only PREMOS-A specific changes, but hold for both instruments as a function of the exposure time. Thus, these ratios can then be assigned to the sensitivity change of PREMOS-B as a function of the exposure time on PREMOS-B. These sensitivity ratios are labelled 7-12 and correspond to the calender dates when PREMOS-B had accumulated exactly the corresponding exposure times of A, i.e. when the ratios $1-6$ have been measured. Thus, we can quantify the sensitivity changes of PREMOS-B between its exposure days 1.91 and 2.50 , and $4.89,8.44$, 10.71, and 12.31, respectively (see Table 1).

First, we identify at what calendar date PREMOS-B had the same exposure times as for when the ratios PREMOSA:PREMOS-B (groups 1-6) were measured. PREMOS-B reached an exposure time of 3.75 days during its time as backup instrument. PREMOS-A reached this exposure time at mission day 44 (solid blue vertical line, Figs. 3a and 3b). The ratios PREMOS-A:PREMOS-B and PREMOS-A in comparison to DIARAD and PMO6-V, in Figure 2, confirm that PREMOS-A and PREMOS-B underwent a very similar level of sensitivity increase by the exposure time of 3.75 days. Groups 1 and 2 in Figure 3 correspond to the only times that PREMOS-A and -B observed simultaneously and for which PREMOS-A had an amount of exposure as PREMOS-B before it became the primary. The ratios are listed in Table 1 , and shown in Figure 3a (plotted as a function of exposure time of PREMOS-A) connected to Figures $3 \mathrm{~b}$ and $3 \mathrm{c}$ (which are plotted in terms of mission time and exposure time of PREMOS-B, respectively) with dashed blue lines. The same exposure times for PREMOS-B occurred at groups 7 and 8, highlighted with red dashed vertical lines in Figures $3 \mathrm{~b}$ and $3 \mathrm{c}$; the same exposure times for ratios 3-6 after 3.75 exposure days correspond to points 9-12 (see also dates listed in Table 1). This is clearer in Figure 3c where the PREMOS-B data in Figure $3 \mathrm{~b}$ are replotted as a function of PREMOS-B exposure time.

The next step is to correct the PREMOS-B data within the exposure periods highlighted by the red groups 7-12 in Figure 3c. In other words, we are aiming to only correct PREMOS-B with known ratios for the dates with corresponding exposure accumulation. We do not apply any fitting procedure to interpolate between ratio dates. To apply this correction appropriately, we must consider that each group at 1-6 contains up to 48 observations (see Table 1); ratios 2 and 3 have an especially large scatter due to the additional heater. We estimate the $81.7 \mathrm{ppm}$ per exposure day sensitivity increase from Schmutz et al. (2013) to have an uncertainty of $10 \%$, or $\sim 8 \mathrm{ppm}$. We use this as a starting point, sampling a random value from this sensitivity increase estimate. We then use bootstrapping (sampling with replacement) to propagate uncertainties on the mean and produce robust error estimates of the mean through $1 \times 10^{6}$ samples; the results correspond with estimates from standard approaches to error analysis, as seen in rows 10 and 11 of Table 1 . Since we are interested in the mean and the uncertainty on this estimate, we do not 
W.T. Ball et al.: PREMOS beginning to end-of-mission sensitivity change assessment

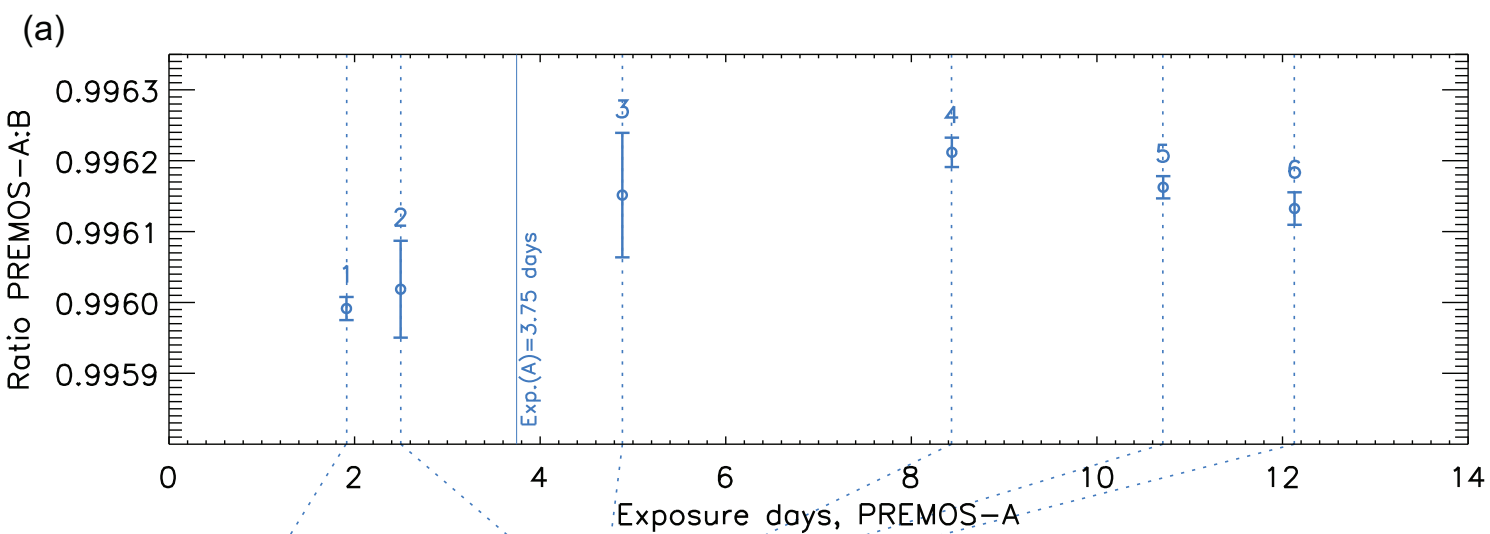

(b-i)
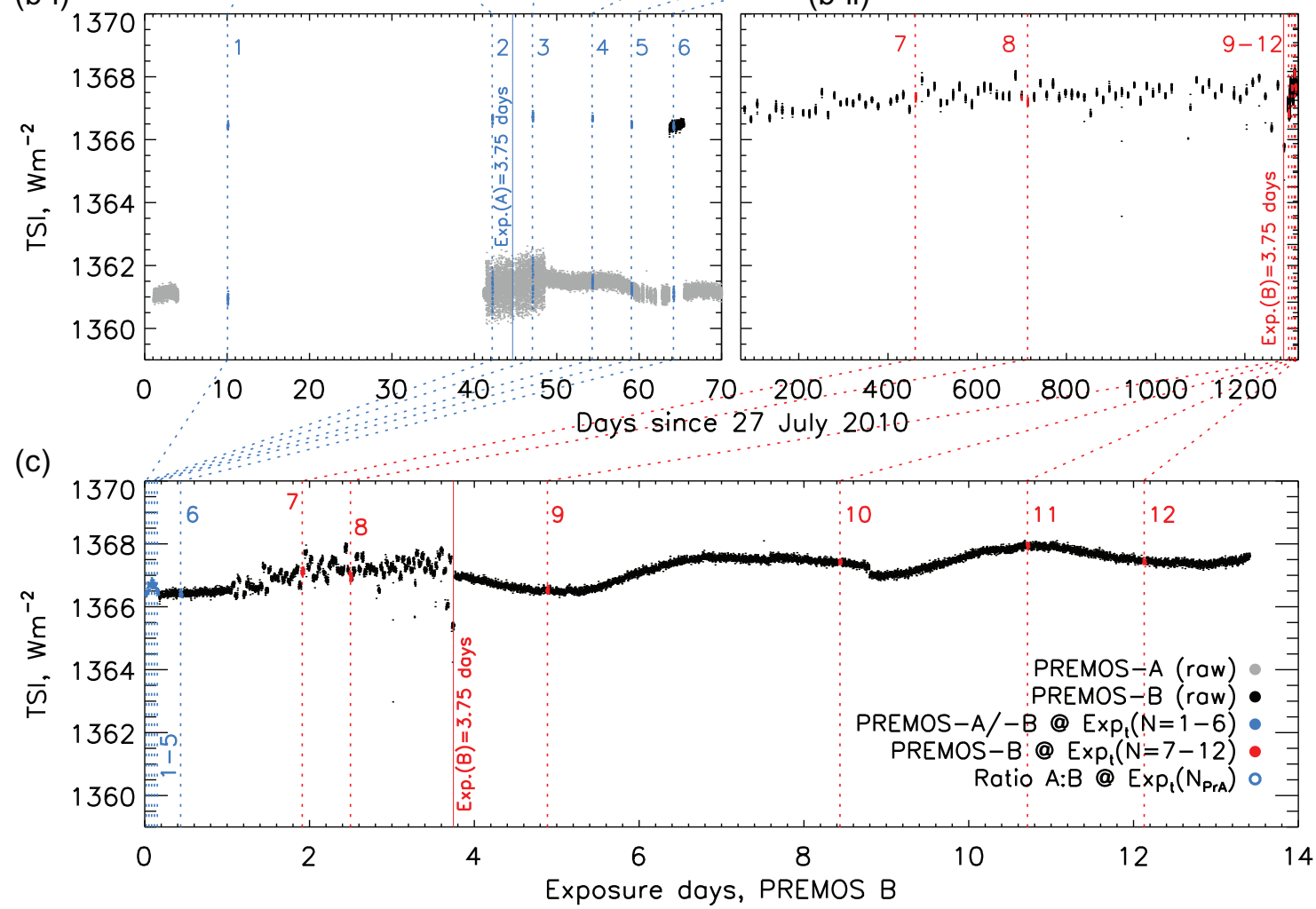

Fig. 3. (a) The ratio PREMOS-A:PREMOS-B during the overlap of observations at groups 1-6, as shown in (b) and linked by the blue, dashed lines. Exposure time is for PREMOS-A. Error bars represent the $95 \%$ confidence interval uncertainty on the mean from bootstrapping. (b) Raw PREMOS-A (grey) and PREMOS-B (black) TSI observations up to mission day 70 (b-i) and PREMOS-B for the full mission up to 1317 days (b-ii), or 27 July 2010 to 4 March 2014. The six periods of co-temporal measurements of A and B are highlighted blue with blue dashed vertical lines and numbered 1-6. Red groups 7-12 and red vertical dashed lines are when PREMOS-B had the same exposure as A at groups 1-6, respectively. (c) PREMOS-B data, as plotted in (b), but in terms of exposure time of PREMOS-B. The ratios labelled 1-6 in (a) are used to correct groups 7-12 here. For clarity, blue dashed lines between (a), (b) and (c) link the ratios/groups 1-6 from exposure-time in terms of PREMOS-A in (a) to mission time in (b) and exposure-time in terms of PREMOS-B in (c); red dashed lines link groups 7-12 in (b) and (c).

propagate the standard deviation uncertainty. The mean, ratios and $95 \%$ confidence intervals are plotted in Figures 2 and $3 \mathrm{a}$. Note that the $68 \%, 95 \%, 99 \%$, etc. confidence intervals calculated from bootstrapping are equal to $1.00,1.96,2.58$, etc. multiples of the standard error on the mean because the sampling distribution is Gaussian.

\section{Estimate of the change in TSI between October 2011 and February 2014}

The resulting corrected PREMOS-B values derived from groups 7 to 12 in Figure 3 and Table 1 are shown in the upper panels of Figure 4 as absolute values shifted by the difference between SOHO/VIRGO and point 7 (red) so they agree on 31 October 2011 , i.e. the date that observations in 7 were made (see Fig. 4a); SOHO/VIRGO is also shifted to SORCE/TIM at this date. Groups $8-12$ are relative to point 7 (Figs. 4b and 4c). Figure 4c shows groups 9-12 in February and March 2014 along with SOHO/VIRGO and SORCE/TIM. SORCE/TIM was not operating during the overlap period with the PREMOS-B values. However, there are SORCE/ TIM observations in December 2013 and late March onwards which have an approximately constant offset relative to SOHO/VIRGO (see Fig. 1). We estimate this offset to be $\sim+0.114 \mathrm{~W} \mathrm{~m}^{-2}$ by calculating a running 81-day median on 
Table 1. (Upper panel) Summary of periods at which ratios of PREMOS-A:PREMOS-B were calculated, and (lower panel) the subsequent relative difference of TSI, in $\mathrm{W} \mathrm{m}^{-2}$, between PREMOS-B and SOHO/VIRGO relative to groups 7 and 8 (see also Fig. 4). All uncertainties are stated for confidence intervals of $68 \%$.

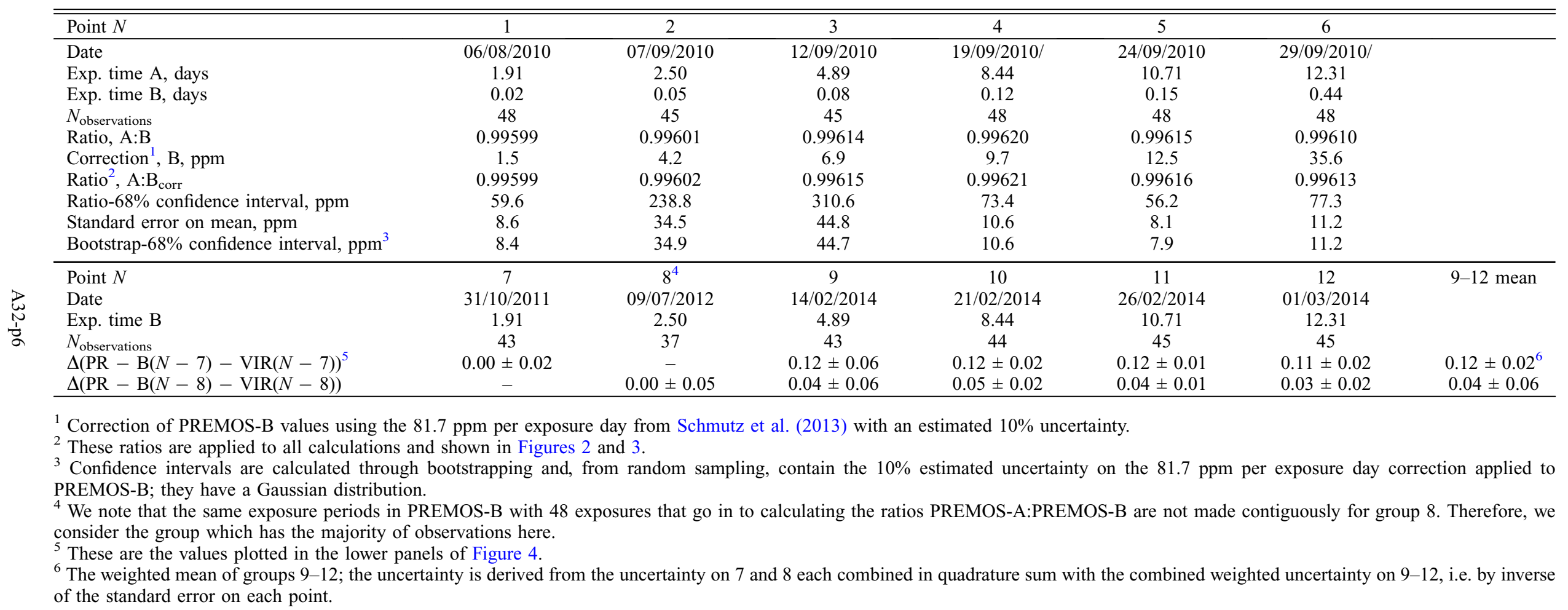



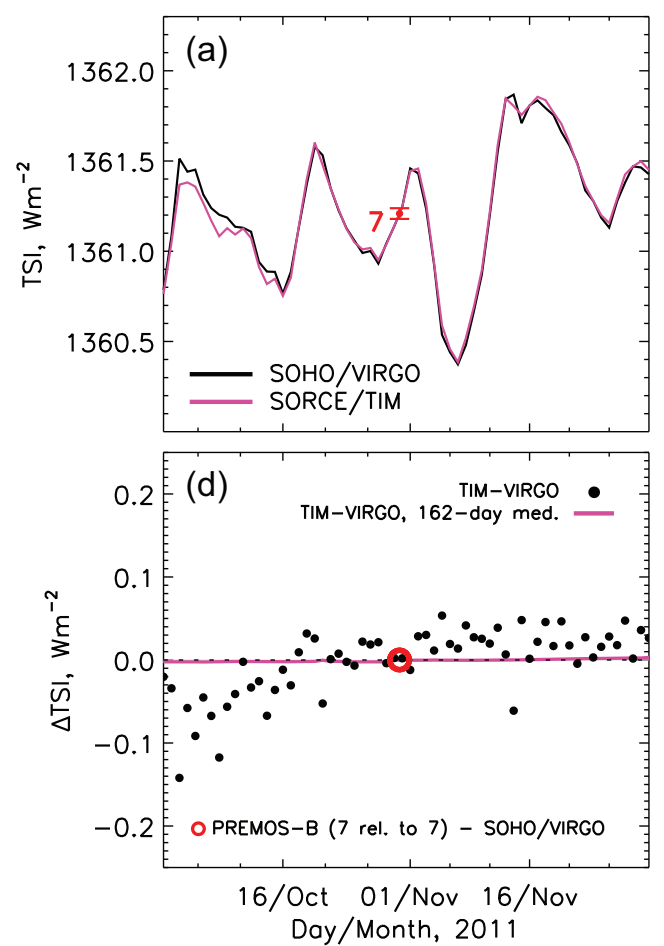
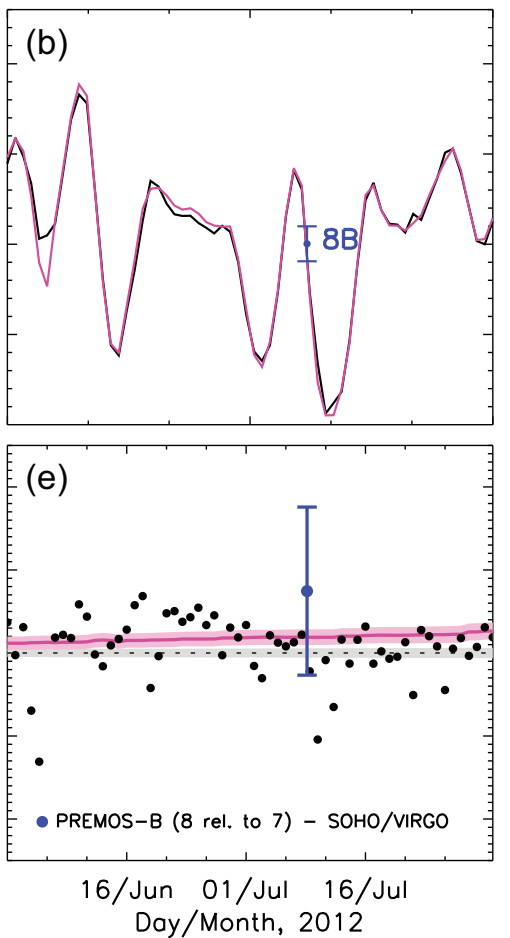
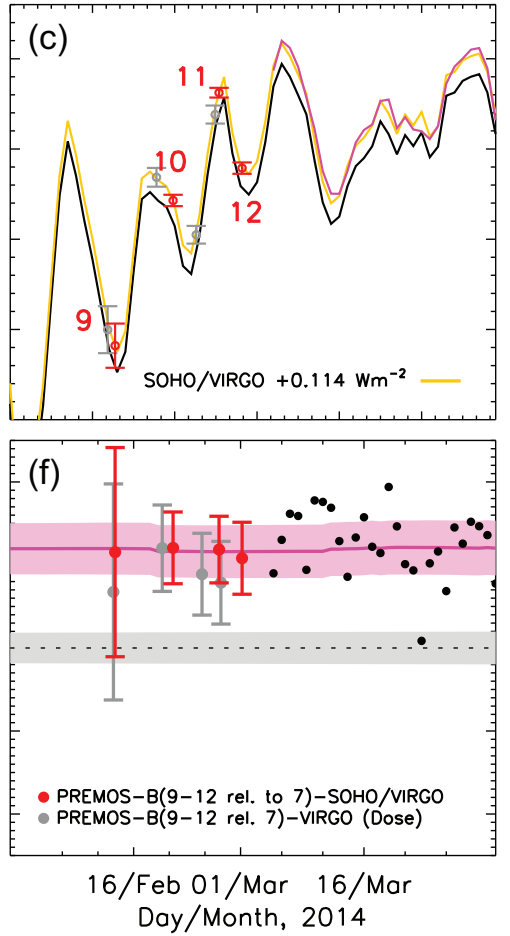

Fig. 4. Two-month periods as highlighted with blue horizontal bars in Figure 2: (a, d) October and November 2011; (b, e) May and June 2012; (c, f) February and March, 2014. (Upper row, a-c) As for the upper plot of Figure 2 with PREMOS-B groups 7-12 also plotted (see Table 1). (Lower row, d-f) As for the lower plot of Figure 2, with PREMOS-B groups 7-12 (see Table 1 also plotted; errors on 7 (red) have been propagated to groups $9-12$ in (f). Error bars in (a)-(c) represent $68 \%$ confidence intervals, and $95 \%$ confidence intervals in (f). Grey dots with error bars represent dose-based estimates of exposure time based on Lyman- $\alpha$.

SORCE/TIM-SOHO/VIRGO (purple line, lower panel of Fig. 1). We, therefore, also plot SOHO/VIRGO shifted by $+0.114 \mathrm{~W} \mathrm{~m}^{-2}$ (yellow) in Figure 4c. PREMOS-B TSI values (red) are closer to SORCE/TIM than SOHO/VIRGO.

To make this clearer, in the lower row of Figure 4, we plot SORCE/TIM (black dots) and PREMOS-B (coloured circles and dots) minus SOHO/VIRGO with an adjustment to SORCE/TIM so that the 81-day-running median and PREMOS-B group 7 cross the zero line at 31 October 2011 (Fig. $4 \mathrm{~d}$ ); Figures $4 \mathrm{c}$ and $4 \mathrm{f}$ are relative to this date. In addition, as for Figure 1, we add a $10 \mathrm{ppm} \mathrm{yr}^{-1}$ range around the SORCE/TIM median line to show the SORCE/TIM stability estimate relative to 31 October 2011. The grey shading represents a $6 \mathrm{ppm} \mathrm{yr}^{-1}$ stability change of SOHO/VIRGO since November 2011, which amounts to 14 ppm (see Footnote 1). To compare the relative change with uncertainties, compared to 31 October 2011, we propagate the uncertainties on the mean of group 7 (red) to groups 9-12 in Figure 4f, which is why the error bars expand on all groups compared to Figure 4c. Figure $4 \mathrm{f}$ shows the overlap period in February and March 2014. We do not show the values resulting from ratios to group 8 because of their larger uncertainty, which does not allow for a conclusive judgement. The alignment of red groups 9-12 with the SORCE/TIM median estimate in Figure 1 is very good. The results clearly indicate that PREMOS-B agrees better with the TSI values of SORCE/TIM. To quantify this, in Table 1 we give the relative difference to SOHO/VIRGO of groups 9-12 relative to groups 7 and 8 . In the last column of Table 1 we calculate the weighted means of the differences in columns 4-7, with uncertainties calculated taking the weighted uncertainty and adding it in quadrature with the uncertainty on groups 7 and 8 . We note that results relative to group 8 have much larger uncertainties than group 7 , which is due to the additional heater being on when ratios 2 and 3 were calculated. The results relative to group 7, with much lower uncertainties and a weighted mean value of $+0.12 \mathrm{~W} \mathrm{~m}^{-2}$, are in better agreement with SORCE/ TIM $\left(+0.114 \mathrm{~W} \mathrm{~m}^{-2}\right)$ than SOHO/VIRGO. This agreement is illustrated in Figure 4, where 95\% confidence intervals on PREMOS-B groups 9-12 clearly agree better with SORCE/TIM.

Finally, we mention that the impact of considering a Lyman- $\alpha$-adjusted dose, instead of simply the exposure time, leads to a small adjustment to the calendar dates when the corresponding dose is reached (grey circles and error bars in Fig. 2). When considered and propagated through to PREMOS-B TSI estimates there is a shift in groups 9-12 (grey points in Figs. $4 \mathrm{c}$ and $4 \mathrm{f}$ ) and a slight decrease in the weighted-mean relative to SOHO/VIRGO $\left(+0.09 \mathrm{~W} \mathrm{~m}^{-2}\right)$. The impact of considering dose is negligible and the result considering this dose-adjusted exposure time has no impact on our conclusion that PREMOS-B agrees with SORCE/ TIM. This implies that our result is robust since Lyman- $\alpha$ represents an extreme adjustment from exposure time only to a Lyman- $\alpha$ weighted dose.

\section{Conclusions}

We have shown that the PREMOS-B radiometer used as a backup reference for the PREMOS TSI instrument degraded in the same way as the primary radiometer, PREMOS-A. This result was achieved by using the currently unique procedure to switch the roles of PREMOS-A and PREMOS-B for 
the remaining 2 weeks of the mission. This revealed that, at 3.75 exposure days, PREMOS-B was at the end of a linear increase during its time as the backup radiometer. As such, the assumed linearity with which to correct PREMOS-B for the entire mission in data release 1 of Schmutz et al. (2013) was reasonable for most of the PREMOS operation. In further investigations we will assess if, towards the end of the PREMOS lifetime, an improvement over a linear correction would be justified. In this work, we focused on the assessment of PREMOS-B exposure days, for which we can directly calculate sensitivity-change ratios using the assumption that PREMOS-B degraded in the same way as PREMOS-A.

PREMOS-B was corrected at exposure times that correspond to the accumulated exposure time of PREMOS-A when PREMOS-A to PREMOS-B ratios have been measured. Following this correction (see Table 1), we use the measured irradiance from PREMOS-B at these times to investigate if SORCE/TIM or SOHO/VIRGO was more likely stable. Relative to October 2011 and February 2014, SORCE/TIM and SOHO/VIRGO differ by $\sim 0.114 \mathrm{~W} \mathrm{~m}^{-2}$. PREMOS-B supports the irradiance values measured by SORCE/TIM.

The full understanding of the sensitivity changes of the backup reference radiometer is critical to successfully estimating the long-term stability of TSI measurements. In the case of PREMOS, our investigation of the backup's final exposure shows that the assumed changes in the backup, based on those in the primary, were valid. Verifying such assumptions is important for assigning an uncertainty estimate to the long term stability of a space radiometer. For PREMOS, we can now confidently assign an error-bar to its October 2011February 2014 stability, which is $0.02 \mathrm{~W} \mathrm{~m}^{-2}$ over 2.3 years (see Table 1), or 6 ppm per year. This in turn, allows for an estimate of the uncertainty of a decadal TSI composite of past years, which is mostly based on SORCE/TIM, whose stability was confirmed by this study. Prior to the last 10 years we rely on VIRGO, for which the stability was not confirmed.

From our examination it is not known whether this drift of VIRGO is systematic or random. Assuming it to be systematic, we extrapolate the disagreement of VIRGO for February 2014, which is $0.12 \mathrm{~W} \mathrm{~m}^{-2}$ or $\sim 90 \mathrm{ppm}$ after 2.3 years, to longer timescales. This gives a stability estimate for VIRGO of $\sim 38 \mathrm{ppm} \mathrm{yr}^{-1}$ or an uncertainty of $460 \mathrm{ppm}$ for a trend estimate between the solar cycle minima of 1996 and 2008: this translates to an uncertainty value of $\sim 0.6 \mathrm{Wm}^{-2}$ for a measured difference between cycle minima. Assuming the $90 \mathrm{ppm}$ disagreement was reached by a random drift process we get about $200 \mathrm{ppm}$ uncertainty for a trend estimate between the solar cycle minima by applying the square root of the ratio of time periods. Thus, we conclude safely that with such uncertainties we are definitely not in a position to assess a potential centennial timescale change in solar irradiance based on any of the presently existing TSI composites. The final goal would be to understand the Sun's impact on natural climate change for which we realize that we still need to continue to monitor TSI in the future with lower uncertainty.

Acknowledgements. We thank the two anonymous referees and Greg Kopp for their thorough input and comments that have improved the paper, and Claus Fröhlich and Till Hoffmann for their helpful and fruitful discussions. We acknowledge the use of data version 6_005_1602 of the VIRGO Experiment on the cooperative ESA/NASA Mission SOHO from the VIRGO Team through PMOD/WRC, Davos, Switzerland. We also acknowledge the use of version 17 of SORCE/TIM TSI data from the NASA SORCE Mission Team. WTB was funded by Swiss National Science Foundation (SNSF) Grant 200020_163206. WS acknowledges support by Projects 200020_153302 and CRSII2-147659 of the SNSF. WF, AF and BW were supported by SNSF Grant Number 200020_116090. The editor thanks two anonymous referees for their assistance in evaluating this paper.

\section{References}

Dewitte, S., D. Crommelynck, S. Mekaoui, and A. Joukoff. Measurement and uncertainty of the long-term total solar irradiance trend. Sol. Phys., 224, 209-216, 2004, DOI: $10.1007 / \mathrm{s} 11207-005-5698-7$.

Fehlmann, A., G. Kopp, W. Schmutz, R. Winkler, W. Finsterle, and N. Fox. Fourth World Radiometric Reference to SI radiometric scale comparison and implications for on-orbit measurements of the total solar irradiance. Metrologia, 49, S34-S38, 2012, DOI: 10.1088/0026-1394/49/2/S34.

Fröhlich, C. Long-term behaviour of space radiometers. Metrologia, 40, 60, 2003, DOI: 10.1088/0026-1394/40/1/314.

Fröhlich, C. Evidence of a long-term trend in total solar irradiance. $A \& A, \mathbf{5 0 1}, \mathrm{L} 27-\mathrm{L} 30,2009$, DOI: $10.1051 / 0004-6361 / 200912318$.

Fröhlich, C. Total solar irradiance: What have we learned from the last three cycles and the recent minimum? Space Sci. Rev., 176(1), 237-252, 2013, DOI: 10.1007/s11214-011-9780-1.

Fröhlich, C., D.A. Crommelynck, C. Wehrli, M. Anklin, S. Dewitte, et al. In-flight performance of the VIRGO solar irradiance instruments on SOHO. Sol. Phys., 175, 267-286, 1997, DOI: $10.1023 / \mathrm{A}: 1004929108864$

Hickey, J.R., B.M. Alton, H.L. Kyle, and D. Hoyt. Total solar irradiance measurements by ERB/Nimbus-7 and a review of nine years. Space Sci. Rev., 48, 321-342, 1988,

DOI: $10.1007 / B F 00226011$.

Kopp, G. An assessment of the solar irradiance record for climate studies. J. Space Weather Space Clim., 4, A14, 2014, DOI: $10.1051 / \mathrm{swsc} / 2014012$

Kopp, G. Magnitudes and timescales of total solar irradiance variability. J. Space Weather Space Clim., 6, A30, 2016, DOI: $10.1051 / \mathrm{swsc} / 2016025$.

Kopp, G., and G. Lawrence. The Total Irradiance Monitor (TIM): Instrument design. Sol. Phys., 230, 91-109, 2005, DOI: $10.1007 / \mathrm{s} 11207-005-7446-4$.

Lee III, R.B., M.A. Gibson, R.S. Wilson, and S. Thomas. Long-term total solar irradiance variability during sunspot cycle 22 . J. Geophys. Res., 100, 1667-1675, 1995, DOI: 10.1029/94JA02897.

Schmutz, W., A. Fehlmann, W. Finsterle, G. Kopp, and G. Thuillier. Total solar irradiance measurements with PREMOS/PICARD. In American Institute of Physics Conference Series, vol. 1531 of American Institute of Physics Conference Series, 624-627, 2013, DOI: $10.1063 / 1.4804847$

Willson, R.C. ACRIM3 and the Total Solar Irradiance database. Astrophys. Space Sci., 352, 341-352, 2014, DOI: $10.1007 / \mathrm{s} 10509-014-1961-4$.

Willson, R.C. and A.V. Mordvinov. Secular total solar irradiance trend during solar cycles 21-23. Geophys. Res. Lett., 30(5), 1199, 2003, DOI: $10.1029 / 2002$ GL016038.

\footnotetext{
Cite this article as: Ball WT, Schmutz W, Fehlmann A, Finsterle W \& Walter B. Assessing the beginning to end-of-mission sensitivity change of the PREcision MOnitor Sensor total solar irradiance radiometer (PREMOS/PICARD). J. Space Weather Space Clim., 6, A32, 2016, DOI: $10.1051 / \mathrm{swsc} / 2016026$.
} 